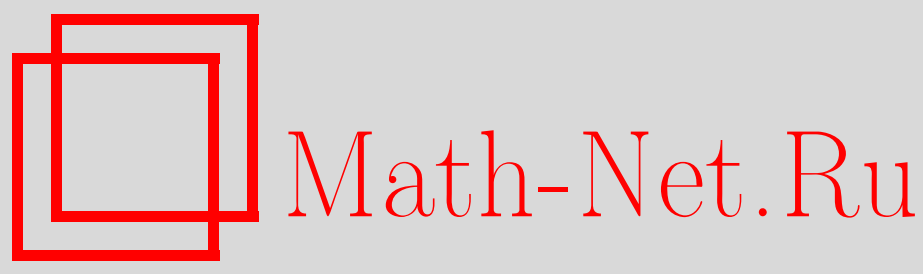

В. Б. Бобров, С. А. Тригер, Конденсат Бозе-Эйнштейна и особенности частотной дисперсии диэлектрической проницаемости в неупорядоченной кулоновской системе, TMФ, 2018, том 194, номер 3, 468-480

DOI: https://doi.org/10.4213/tmf9283

Использование Общероссийского математического портала Math-Net.Ru подразумевает, что вы прочитали и согласны с пользовательским соглашением http://www.mathnet.ru/rus/agreement

Параметры загрузки:

IP: 3.89 .185 .249

26 апреля 2023 г., 16:09:01

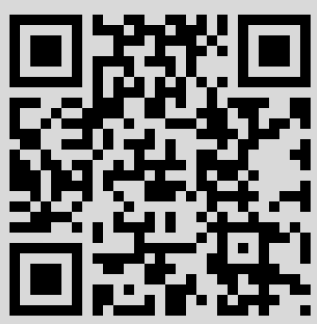




\title{
КОНДЕНСАТ БОЗЕ-ЭЙНШТЕЙНА И ОСОБЕННОСТИ ЧАСТОТНОЙ ДИСПЕРСИИ ДИЭЛЕКТРИЧЕСКОЙ ПРОНИЦАЕМОСТИ В НЕУПОРЯДОЧЕННОЙ КУЛОНОВСКОЙ СИСТЕМЕ
}

\begin{abstract}
В рамках теории линейного отклика рассмотрена частотная дисперсия диэлектрической проницаемости неупорядоченной кулоновской системы при наличии одночастичного конденсата Бозе-Эйнштейна для ядер. Показано, что в такой системе имеет место сверхпроводимость ядер, которая проявляется в эффекте Мейсснера для слабонеоднородного электромагнитного поля низкой частоты. Полученный результат предоставляет возможность для решения вопроса о наличии одночастичного конденсата Бозе-Эйнштейна в сверхтекучем Не-ІІ на основе прямых экспериментов.
\end{abstract}

Ключевые слова: конденсат Бозе-Эйнштейна, кулоновская система, диэлектрическая проницаемость, частотная дисперсия.

DOI: https://doi.org/10.4213/tmf9283

\section{1. ВВЕДЕНИЕ}

Принятая в настоящее время микроскопическая модель для сверхтекучего Не-ІІ представляет собой систему нейтральных атомов-бозонов с нулевым спином, которые образуют конденсат Бозе-Эйнштейна (КБЭ) - макроскопическое число частиц в состоянии с нулевым импульсом [1], [2]. При этом предполагается, что атомы гелия взаимодействуют между собой посредством некоторого короткодействующего эффективного потенциала, вид которого, строго говоря, неизвестен в силу большой плотности, однако имеет существенное значение при описании сверхтекучего Не-II [3]. С другой стороны, результаты недавних экспериментов [4], [5] показывают электрическую активность сверхтекучего Не-II, что подразумевает рассмотрение

Работа выполнена при финансовой поддержке Российского научного фонда (грант № 14-50-00124).

* Объединенный институт высоких температур РАН, Москва, Россия. E-mail: vic5907@mail.ru, satron@mail.ru

${ }^{\dagger}$ Национальный исследовательский университет "Московский энергетический институт", Москва, Россия 
нейтрального атома гелия как составной частицы, представляющей собой связанное состояние ядра и электронов [6], [7]. Это приводит к необходимости исследования электромагнитных свойств нейтральной жидкости как совокупности нерелятивистских электронов и ядер, взаимодействующих между собой по закону Кулона (кулоновской системы (KC)) [8]. Такой подход позволяет единым образом описывать как диэлектрики, в которых электроны и ядра находятся преимущественно в связанных (атомарных, молекулярных и т. п.) состояниях, так и проводники, в которых существенное значение имеют состояния рассеяния заряженных частиц (см. подробнее [9] и цитированную там литературу). Разделение веществ на диэлектрики и проводники определяется термодинамическими параметрами и носит условный характер, обусловленный существенным количественным различием в значениях статической проводимости. При этом представление об атомарных, молекулярных и иных связанных состояниях электронов и ядер имеет смысл только в адиабатическом приближении (приближении Борна-Оппенгеймера) для подсистемы ядер как в квантовой механике [10], так и в квантовой статистике [11]. Отметим, что применение кулоновской модели вещества для описания области термодинамических параметров, которые характеризуются наличием одночастичного КБЭ, находится в начальной стадии разработки [12]. В настоящей работе рассмотрены особенности частотной дисперсии диэлектрической проницаемости (ДП) для неупорядоченной КС при наличии одночастичного КБЭ. Использование теории линейного отклика и общего определения для одночастичного КБЭ позволяет получить результаты вне рамок теории возмущений по кулоновскому взаимодействию между заряженными частицами.

\section{2. ОДНОЧАСТИЧНЫЙ КБЭ И НЕДИАГОНАЛЬНЫЙ ДАЛЬНИЙ ПОРЯДОК В ОДНОЧАСТИЧНОЙ МАТРИЦЕ ПЛОТНОСТИ}

Согласно существующим представлениям возможность существования одночастичного КБЭ обусловлена тождественностью исходных частиц-бозонов. Таковыми в рамках кулоновской модели могут быть только ядра, которые в нерелятивистском приближении рассматриваются нами как точечные (не составные) частицы. Необходимо подчеркнуть, что кулоновское взаимодействие между заряженными частицами не может изменить статистику частиц. Другими словами, коммутационные соотношения для полевых операторов исходных заряженных частиц (электронов и ядер) не зависят от взаимодействия между частицами. Тем самым образование КБЭ ядер, вообще говоря, возможно как для диэлектриков, так и для проводников. При этом в настоящее время отсутствует микроскопическое обоснование для модельной многочастичной системы "атомов", использование которой исторически сложилось при описании инертных (диэлектрических) жидкостей, включая сверхтекучий Не-II.

Решение вопроса об определении термодинамических параметров, при которых образуется КБЭ в КС, не представляется возможным. Поэтому мы будем рассматривать систему взаимодействующих электронов и ядер, предполагая, что ядра (величины, соответствующие ядрам, помечены ниже индексом с) являются бозонами, что и приводит к появлению одночастичного КБЭ для ядер при низких температурах. При этом электроны, будучи фермионами, не образуют одночастичный КБЭ, но влияют на значения термодинамических параметров, при которых появляется 
одночастичный КБЭ ядер. Описание КБЭ для так называемых составных частиц требует отдельного обсуждения (см. ниже).

Согласно общему определению, которое было предложено Пенроузом и Онзагером [13], наличие КБЭ связано с аномальным пространственным поведением равновесной одночастичной матрицы плотности и получило название недиагонального дальнего порядка (НДДП) [14]. Это утверждение для однородной и изотропной КС, в которой одночастичная матрица плотности ядер имеет вид $\gamma_{\mathrm{c}}\left(\mathbf{r}, \mathbf{r}^{\prime}\right)=\gamma_{\mathrm{c}}\left(\left|\mathbf{r}-\mathbf{r}^{\prime}\right|\right)$, можно записать как

$$
\lim _{\left|\mathbf{r}-\mathbf{r}^{\prime}\right| \rightarrow \infty} \gamma_{\mathrm{c}}\left(\mathbf{r}, \mathbf{r}^{\prime}\right)=n_{\mathrm{c}}^{\mathrm{BEC}} \neq 0, \quad \gamma_{\mathrm{c}}\left(\mathbf{r}, \mathbf{r}^{\prime}\right) \equiv\left\langle\widehat{\Psi}_{\mathrm{c}}^{+}(\mathbf{r}) \widehat{\Psi}_{\mathrm{c}}\left(\mathbf{r}^{\prime}\right)\right\rangle,
$$

где $n_{\mathrm{c}}^{\text {вес }}-$ плотность числа ядер в одночастичном КБЭ, $\widehat{\Psi}_{\mathrm{c}}^{+}(\mathbf{r})$ и $\widehat{\Psi}_{\mathrm{c}}(\mathbf{r})-$ соответственно полевые операторы рождения и уничтожения для ядер, угловые скобки означают усреднение с распределением Гиббса, которое определяется точным гамильтонианом для нерелятивистской системы взаимодействующих электронов и ядер. В нормальной $\mathrm{KC} n_{\mathrm{c}}^{\mathrm{BEC}}=0$, т. е. КБЭ отсутствует.

Согласно сказанному выше при использовании определения (1) наличие КБЭ в $\mathrm{KC}$ может быть связано только с ядрами-бозонами. Отметим, что КC, находящаяся в сверхпроводящем состоянии для электронов, характеризуется наличием НДДП для двухчастичной матрицы плотности электронов [14].

Для обеспечения термодинамического равновесия в $\mathrm{KC}$ должно выполняться условие квазинейтральности: $\sum_{a=\mathrm{e}, \mathrm{c}} z_{a} e n_{a}=0$, где $n_{a}=\left\langle\widehat{N}_{a}\right\rangle / V-$ средняя плотность для числа частиц сорта $а$ с зарядом $z_{a} e$ и массой $m_{a}$ в объеме $V, \widehat{N}_{a}=$ $\int d \mathbf{r} \widehat{\Psi}_{a}^{+}(\mathbf{r}) \widehat{\Psi}_{a}(\mathbf{r})$ - оператор полного числа частиц сорта $a$ [8]. Электронам соответствует индекс е.

Необходимо также учитывать, что в статистической теории усреднение с распределением Гиббса соответствует состоянию термодинамического равновесия только после перехода к термодинамическому пределу: $\left\langle\widehat{N}_{a}\right\rangle \rightarrow \infty, V \rightarrow \infty, n_{a}=\left\langle\widehat{N}_{a}\right\rangle / V=$ const. Это означает, что при вычислении соответствующих средних величин необходимо первоначально рассматривать систему в очень большом (макроскопическом), но конечном объеме $V$, а затем осуществить термодинамический предельный переход [15].

Для осуществления такого предельного перехода в (1) используем дискретное импульсное представление для полевых операторов $\widehat{\Psi}_{\mathrm{c}}^{+}(\mathbf{r})$ и $\widehat{\Psi}_{\mathrm{c}}(\mathbf{r})[15]$. В результате одночастичную матрицу плотности для ядер в однородной и изотропной КС можно представить в виде ряда Фурье [15]:

$$
\gamma_{\mathrm{c}}\left(\left|\mathbf{r}-\mathbf{r}^{\prime}\right|\right)=\frac{\left.f_{\mathrm{c}}^{(V)}\right|_{\mathbf{p}=0}}{V}+\frac{1}{V} \sum_{\mathbf{p} \neq 0} f_{\mathrm{c}}^{(V)}(\mathbf{p}) e^{i \mathbf{p} \cdot\left(\mathbf{r}^{\prime}-\mathbf{r}\right)},
$$

где $f_{\mathrm{c}}^{(V)}(\mathbf{p})=\left\langle\hat{c}_{\mathbf{p}}^{+} \hat{c}_{\mathbf{p}}\right\rangle^{(V)}$ - среднее число заполнения ядер в состоянии с импульсом $\hbar \mathbf{p}$ (или одночастичная функция распределения по импульсам), индекс $(V)$ означает, что соответствующая функция отвечает системе в очень большом (макроскопическом), но конечном объеме $V, \hat{c}_{\mathbf{p}}^{+}$и $\hat{c}_{\mathbf{p}}$ - соответственно операторы рождения и уничтожения для ядер с импульсом $\hbar \mathbf{p}$ (здесь и далее полагаем, что ядра обладают нулевым спином). 
При записи (2) учитывается, что объем системы достаточно велик, поэтому можно пренебречь граничными эффектами, приводящими к неоднородности. В этом случае одночастичная матрица плотности $\gamma_{\mathrm{c}}\left(\mathbf{r}, \mathbf{r}^{\prime}\right)$ может быть представлена как $\gamma_{\mathrm{c}}\left(\left|\mathbf{r}-\mathbf{r}^{\prime}\right|\right)$.

Согласно (1), (2) средняя плотность числа ядер в одночастичном КБЭ равна

$$
n_{\mathrm{c}}^{\mathrm{BEC}}=\frac{\left.f_{\mathrm{c}}^{(V)}\right|_{\mathbf{p}=0}}{V} .
$$

Это означает, что среднее число заполнения ядер с нулевым импульсом $\left\langle\widehat{N}_{0}\right\rangle=$ $\left.f_{\mathrm{c}}^{(V)}\right|_{\mathbf{p}=0} \equiv\left\langle\hat{c}_{0}^{+} \hat{c}_{0}\right\rangle=n_{\mathrm{c}}^{\text {Вес }} V$ является макроскопической величиной, что и является определением КБЭ.

После перехода к термодинамическому пределу функция распределения по импульсам для ядер при наличии КБЭ имеет вид

$$
f_{\mathrm{c}}(\mathbf{p})=\left\langle\widehat{N}_{0}\right\rangle \delta_{\mathbf{p}, 0}+f_{\mathrm{c}}^{\text {(over) }}(p)\left(1-\delta_{\mathbf{p}, 0}\right),
$$

где $f_{\mathrm{c}}^{\text {(over) }}(p)$ - одночастичная функция распределения для ядер в надконденсатном состоянии при $\mathbf{p} \neq 0$.

При этом средняя плотность числа ядер $n_{\mathrm{c}} \equiv\left\langle\widehat{N}_{\mathrm{c}}\right\rangle / V$ равна

$$
n_{\mathrm{c}}=\lim _{V \rightarrow \infty} V^{-1} \sum_{\mathbf{p}} f_{\mathrm{c}}^{(V)}(\mathbf{p})=n_{\mathrm{c}}^{\mathrm{BEC}}+n_{\mathrm{c}}^{(\text {over })},
$$

где $n_{\mathrm{c}}^{\text {(over) }}-$ плотность числа ядер в надконденсатных состояниях

$$
n_{\mathrm{c}}^{\text {(over) }}=\lim _{V \rightarrow \infty} V^{-1} \sum_{\mathbf{p} \neq 0} f_{\mathrm{c}}^{(V)}(\mathbf{p})=\int \frac{d^{3} p}{(2 \pi)^{3}} f_{\mathrm{c}}^{(\text {over })}(p) .
$$

Таким образом, при наличии одночастичного КБЭ необходимо до перехода к термодинамическому пределу выделить сингулярные члены, соответствующие макроскопическому числу ядер в КБЭ. Аналогичное утверждение имеет место и при рассмотрении неоднородной системы с КБЭ [16].

\section{3. ЧАСТОТНАЯ ДИСПЕРСИЯ ДП И ВРЕМЕННЫ́Е КОРРЕЛЯЦИОННЫЕ ФУНКЦИИ В НОРМАЛЬНОЙ КС}

Прежде чем исследовать влияние одночастичного КБЭ, рассмотрим особенности частотной дисперсии ДП в нормальной КC, находящейся в слабом внешнем электромагнитном поле.

Электромагнитные свойства однородной и изотропной $\mathrm{KC}$ при воздействии на нее слабого электромагнитного поля полностью определяются тензором ДП

$$
\varepsilon_{\alpha \beta}(\mathbf{q}, \omega)=\left(\delta_{\alpha, \beta}-\frac{q_{\alpha} q_{\beta}}{q^{2}}\right) \varepsilon^{\operatorname{tr}}(q, \omega)+\frac{q_{\alpha} q_{\beta}}{q^{2}} \varepsilon^{\mathrm{l}}(q, \omega),
$$

где $\varepsilon^{\operatorname{tr}}(q, \omega)$ и $\varepsilon^{\mathrm{l}}(q, \omega)$ - соответственно поперечная и продольная ДП, учитывающие пространственную и частотную дисперсию. Функции $\varepsilon^{\mathrm{l}}(q, \omega)$ и $\varepsilon^{\operatorname{tr}}(q, \omega)$ в длинноволновом пределе $q \rightarrow 0$ при ненулевых значениях частоты $\omega$ удовлетворяют следующему равенству [17]:

$$
\lim _{q \rightarrow 0} \varepsilon^{\mathrm{l}}(q, \omega)=\lim _{q \rightarrow 0} \varepsilon^{\operatorname{tr}}(q, \omega)=\varepsilon(\omega), \quad \varepsilon(\omega)=1+\frac{4 \pi i}{\omega} \sigma(\omega) .
$$


Теоретическому исследованию частотной дисперсии ДП $\varepsilon(\omega)$ и проводимости $\sigma(\omega)$ уделяется большое внимание (см., например, [18], [19] и цитированную там литературу). При этом непосредственно измеряемая статическая проводимость $\sigma_{\text {st }}$ определяется предельным соотношением

$$
\sigma_{\mathrm{st}}=\lim _{\omega \rightarrow 0} \sigma(\omega)>0
$$

при ненулевой температуре. Таким образом, согласно (8), (9) ДП $\varepsilon(\omega)$ для КС в нормальном состоянии в статическом пределе $\omega \rightarrow 0$ имеет особенность первого порядка [17]:

$$
\left.\varepsilon(\omega)\right|_{\omega \rightarrow 0} \rightarrow \frac{4 \pi i}{\omega} \sigma_{\text {st }} .
$$

При вычислении функции $\varepsilon(\omega)$ используется теория линейного отклика, в рамках которой продольная $\varepsilon^{\mathrm{l}}(q, \omega)$ и поперечная $\varepsilon^{\operatorname{tr}}(q, \omega)$ ДП определяются соответствующими запаздывающими равновесными функциями Грина (обобщенными восприимчивостями), которые заданы при действительных частотах $\omega$ [20], [21]. Отличительной особенностью обобщенной восприимчивости $\chi(\omega)$ является возможность ее представления в виде временно́го интеграла (см., например, [22])

$$
\chi(\omega)=\int_{0}^{\infty} d t e^{i \omega t} f_{\chi}(t),
$$

где временна́я корреляционная функция $f_{\chi}(t)$ удовлетворяет условию

$$
\lim _{t \rightarrow \infty} f_{\chi}(t)=0
$$

которое обеспечивает сходимость интеграла в (11) для произвольных действительных частот $\omega$. Исключением, вообще говоря, является точка $\omega=0$ (см. (10)).

Согласно (11) обобщенная восприимчивость (запаздывающая функция Грина) $\chi(\omega)$ является аналитической функцией в верхней полуплоскости комплексных частот $z$ и, следовательно, удовлетворяет дисперсионным соотношениям (соотношениям Крамерса-Кронига) [21], [22]. При этом значения запаздывающих функций Грина совпадают со значениями соответствующих температурных функций Грина при $\hbar z=i \Omega_{n}, \Omega_{n}=2 \pi n T, n=1,2, \ldots$. Здесь $T$ - температура равновесной системы в энергетических единицах [23].

Для вычисления температурных функций Грина существует хорошо разработанная температурная диаграммная техника теории возмущений [8], [23]. На этой основе определяются функции $\varepsilon^{\mathrm{l}}\left(q, i \Omega_{n}\right)$ и $\varepsilon^{\mathrm{tr}}\left(q, i \Omega_{n}\right)$ в длинноволновом пределе $q \rightarrow 0$ [24], [25]. Затем осуществляется процедура аналитического продолжения с дискретного множества точек на мнимой оси $i \Omega_{n}$ на верхнюю полуплоскость комплексных $z$. В рамках такой процедуры с использованием диаграммной техники теории возмущений вычисляется интересующая нас функция в верхней полуплоскости комплексных частот $z$ [23].

Для определения найденной таким образом величины как функции действительных частот $\omega$ используется следующий прием, который принято называть правилом обхода полюсов Ландау (см., например, [26]):

$$
\varepsilon(\omega)=\lim _{\delta \rightarrow+0} \varepsilon(\omega+i \delta) .
$$


Этот математический прием получил широкое распространение в теории функций Грина в статистической механике [21]. Если найденная функция, заданная в верхней полуплоскости комплексных значений $z$, может быть представлена в виде (11), в реализации процедуры (13) уже нет необходимости. В этом случае достаточно осуществить замену комплексной величины $z$ на действительное значение частоты $\omega$ [27].

В частности, для определения ДП $\varepsilon(\omega)(8)$ используем общее выражение для функции $\varepsilon(z)$, которая является аналитическим продолжением функций $\varepsilon^{\mathrm{l}}\left(q, i \Omega_{n}\right)$ и $\varepsilon^{\operatorname{tr}}\left(q, i \Omega_{n}\right)$ в длинноволновом пределе $q \rightarrow 0$ с дискретного множества точек на мнимой оси на верхнюю полуплоскость комплексных значений $z(\operatorname{Im} z>0)$ [24], [25]

$$
\begin{gathered}
\varepsilon(z)=1-\frac{\omega_{\mathrm{p}}^{2}}{z^{2}}-\frac{\varphi(z)}{z^{2}}, \quad \varphi(z)=\frac{4 \pi}{3}\left\langle\left\langle\hat{I}^{\beta} \mid \hat{I}^{\beta}\right\rangle\right\rangle_{z}, \\
\langle\langle\hat{A} \mid \hat{B}\rangle\rangle_{z} \equiv-\frac{i}{\hbar V} \int_{0}^{\infty} d t e^{i z t}\langle[\hat{A}(t), \widehat{B}(0)]\rangle,
\end{gathered}
$$

где $\omega_{\mathrm{p}}$ - плазменная частота, определяемая средними плотностями чисел электронов и ядер в $\mathrm{KC}: \omega_{\mathrm{p}}^{2}=\sum_{a} \omega_{a}^{2}, \omega_{a}=\left(4 \pi z_{a}^{2} e^{2} n_{a} / m_{a}\right)^{1 / 2}$ - плазменная частота для частиц сорта $a, \hat{I}^{\beta}=\sum_{a} z_{a} e \hat{I}_{a}^{\beta}-$ оператор полного электрического тока,

$$
\hat{I}_{a}^{\beta}=-i \hbar \int d \mathbf{r} \frac{\widehat{\Psi}_{a}^{+}(\mathbf{r}) \nabla_{\mathbf{r} \beta} \widehat{\Psi}_{a}(\mathbf{r})-\nabla_{\mathbf{r} \beta} \widehat{\Psi}_{a}^{+}(\mathbf{r}) \widehat{\Psi}_{a}(\mathbf{r})}{2 m_{a}}
$$

- оператор полного потока числа частиц сорта $a, \hat{A}(t)=e^{i \widehat{H} t / \hbar} \hat{A} e^{-i \widehat{H} t / \hbar}, \widehat{H}-$ точный гамильтониан КС в нерелятивистском приближении. Здесь и далее подразумевается суммирование по повторяющимся индексам. Соотношения (14), (15) следует понимать в термодинамическом пределе.

Далее обратим внимание, что согласно (15) функция $\varphi(z)(14)$ может быть представлена в виде временно́го интеграла (11). Следовательно, эта функция может быть определена при действительных частотах $\omega$ в виде

$$
\varphi(\omega)=\int_{0}^{\infty} d t e^{i \omega t} f_{\varphi}(t), \quad f_{\varphi}(t)=-\frac{4 \pi i}{3 \hbar V}\left\langle\left[\hat{I}^{\beta}(t), \hat{I}^{\beta}(0)\right]\right\rangle,
$$

где временна́я корреляционная функция $f_{\varphi}(t)$ удовлетворяет условию $(12)$.

Таким образом, с учетом соотношений (14)-(16) ДП $\varepsilon(\omega)$ однородной и изотропной $\mathrm{KC}$ при действительных частотах $\omega$ определяется соотношением (14) посредством замены комплексной величины $z$ на действительную величину $\omega$. Отметим, что это утверждение имеет смысл только после перехода в определении временно́й функции $f_{\varphi}(t)(16)$ к термодинамическому пределу [27].

Учтем далее, что

$$
\frac{d \widehat{P}^{\beta}(t)}{d t}=\hat{I}^{\beta}(t), \quad f_{\varphi}(t)=4 \pi \frac{d f_{\sigma}(t)}{d t}, \quad f_{\sigma}(t)=-\frac{i}{3 \hbar V}\left\langle\left[\widehat{P}^{\beta}(t), \hat{I}^{\beta}(0)\right]\right\rangle,
$$

где

$$
\widehat{P}^{\beta}=\sum_{a} z_{a} e \widehat{P}_{a}^{\beta}, \quad \widehat{P}_{a}^{\beta}=\int d^{3} r r_{\beta} \widehat{\Psi}_{a}^{+}(\mathbf{r}) \widehat{\Psi}_{a}(\mathbf{r})
$$

- оператор дипольного момента для частиц сорта $a$. 
Используя (17), выполним в (14) интегрирование по частям:

$$
\begin{gathered}
\varepsilon(\omega)=1-\frac{\omega_{\mathrm{p}}^{2}-\Omega_{\mathrm{p}}^{2}}{\omega^{2}}+\frac{4 \pi i}{\omega} \Phi(\omega), \\
\Phi(\omega)=\int_{0}^{\infty} d t e^{i \omega t} f_{\sigma}(t), \quad \Omega_{\mathrm{p}}^{2}=\frac{4 \pi i}{3 \hbar V}\left\langle\left[\hat{I}^{\beta}(0), \hat{P}^{\beta}(0)\right]\right\rangle .
\end{gathered}
$$

Отметим, что соотношения (18), (19) справедливы при условии

$$
\lim _{t \rightarrow \infty} f_{\sigma}(t)=0
$$

причем, как следует из сказанного выше, переход к пределу $t \rightarrow \infty$ осуществляется после термодинамического предельного перехода в определении (17) для временно́й корреляционной функции $f_{\sigma}(t)[27]$.

Для последовательного перехода к термодинамическому пределу при вычислении величины $\Omega_{\mathrm{p}}^{2}(19)$ необходимо использовать импульсное представление для операторов $\hat{I}_{a}^{\beta}$ и $\widehat{P}_{a}^{\beta}$

$$
\hat{I}_{a}^{\beta}=\sum_{s} \sum_{\mathbf{p} \neq 0} \frac{\hbar p_{\beta}}{m_{a}} \hat{a}_{\mathbf{p} s}^{+} \hat{a}_{\mathbf{p} s}, \quad \widehat{P}_{a}^{\beta}=i \sum_{s} \sum_{\mathbf{p} \neq 0} \hat{a}_{\mathbf{p} s}^{+} \nabla_{\mathbf{p} \beta} \hat{a}_{\mathbf{p} s}
$$

Обратим внимание, что в операторах $\hat{I}_{a}^{\beta}$ и $\widehat{P}_{a}^{\beta}$ в (21) отсутствует вклад, отвечающий нулевому импульсу. Используя далее коммутационные соотношения для операторов рождения $\hat{a}_{\mathbf{p} s}^{+}$и уничтожения $\hat{a}_{\mathbf{p} s}$ частиц сорта $a$ с импульсом $\hbar \mathbf{p}$ и проекцией спина $s$, находим

$$
\left\langle\left[I^{\beta}(0), P^{\beta}(0)\right]\right\rangle=-3 i \hbar \sum_{s} \sum_{\mathbf{p} \neq 0} f_{a}^{(V)}(\mathbf{p}, s), \quad f_{a}^{(V)}(\mathbf{p}, s) \equiv\left\langle\hat{a}_{\mathbf{p} s}^{+} \hat{a}_{\mathbf{p} s}\right\rangle^{(V)} .
$$

Таким образом, для нормальной КС, в которой отсутствует одночастичный КБЭ, согласно (19), (22) имеет место равенство

$$
\Omega_{\mathrm{p}}^{2}=\omega_{\mathrm{p}}^{2}
$$

Подставляя $(23)$ в (18) и учитывая соотношение (8), связывающее ДП $\varepsilon(\omega)$ и проводимость $\sigma(\omega)$, находим

$$
\sigma(\omega)=\Phi(\omega)=\int_{0}^{\infty} d t e^{i \omega t} f_{\sigma}(t)
$$

при условии, что временна́я корреляционная функция $f_{\sigma}(t)$ удовлетворяет условию (20). Это означает, что при указанных условиях частотная дисперсия проводимости $\sigma(\omega)$ нормальной КС при действительных частотах $\omega$ определяется известной формулой Кубо [28].

Введем в рассмотрение еще одну временну́ю корреляционную функцию $f_{\varepsilon}(t)$, понимаемую в термодинамическом пределе,

$$
f_{\varepsilon}(t)=-\frac{i}{3 \hbar V}\left\langle\left[P^{\beta}(t), P^{\beta}(0)\right]\right\rangle .
$$


Из спектрального представления нетрудно убедиться, что функция $f_{\varepsilon}(t)$ принимает действительные значения и согласно (10), (11) удовлетворяет уравнению

$$
\frac{d f_{\varepsilon}(t)}{d t}=f_{\sigma}(t), \quad f_{\varepsilon}(0)=0 .
$$

Если для функции $f_{\varepsilon}(t)$ справедливо условие $(12)$, т. е.

$$
\lim _{t \rightarrow \infty} f_{\varepsilon}(t)=0
$$

то, выполнив в (24) интегрирование по частям, с учетом (8), (26), (27) получаем

$$
\varepsilon(\omega)=1+4 \pi \alpha(\omega), \quad \alpha(\omega)=\int_{0}^{\infty} d t e^{i \omega t} f_{\varepsilon}(t)
$$

где функция $\alpha(\omega)$ имеет смысл поляризуемости для нормальной $\mathrm{KC}$.

Однако соотношение (28) отвечает только состоянию "истинного" диэлектрика, в котором $\mathrm{KC}$ имеет нулевую статическую проводимость: $\sigma_{\mathrm{st}}=0$ [29]. Тем самым в состоянии "истинного" диэлектрика частотная дисперсия ДП $\varepsilon(\omega)$ не соответствует предельному условию (10), но удовлетворяет соотношениям Крамерса-Кронига в традиционной для диэлектриков форме [30]

$$
\operatorname{Re} \varepsilon(\omega)=1+\frac{1}{\pi} \mathrm{P} \int_{-\infty}^{\infty} d \xi \frac{\operatorname{Im} \varepsilon(\xi)}{\xi-\omega}, \quad \operatorname{Im} \varepsilon(\omega)=-\frac{1}{\pi} \mathrm{P} \int_{-\infty}^{\infty} d \xi \frac{\operatorname{Re} \varepsilon(\xi)-1}{\xi-\omega},
$$

где символ Р перед знаками интегралов означает, что соответствующий интеграл понимается в смысле главного значения.

Состояние "истинного" диэлектрика является предельным состоянием КС, peaлизация которого возможна только в предельном случае нулевой температуры или в критической точке (подробнее см. [31], [32] и цитированную там литературу). Это означает, что в КС (за исключением указанных выше предельных случаев) условие $(27)$ для функции $f_{\varepsilon}(t)$ не выполняется [33].

По этой причине для корректного интегрирования по частям в $(24)$ введем в рассмотрение функцию

$$
f_{\varepsilon}^{0}(t)=f_{\varepsilon}(t)-f_{\varepsilon}(\infty), \quad f_{\varepsilon}(\infty)=\lim _{t \rightarrow \infty} f_{\varepsilon}(t), \quad f_{\varepsilon}^{0}(0)=-f_{\varepsilon}(\infty) .
$$

Функция $f_{\varepsilon}^{0}(t)$ удовлетворяет дифференциальному уравнению (26), но с другим начальным условием (30). При этом для функции $f_{\varepsilon}^{0}(t)$ условие (12) строго выполняется:

$$
\lim _{t \rightarrow \infty} f_{\varepsilon}^{0}(t)=0
$$

С учетом (26), (30), (31) интегрирование по частям в (24) приводит к следующему результату:

$$
\varepsilon(\omega)=\varepsilon^{0}(\omega)+\frac{4 \pi i}{\omega} f_{\varepsilon}(\infty), \quad \varepsilon^{0}(\omega)=1+4 \pi \int_{0}^{\infty} d t e^{i \omega t} f_{\varepsilon}^{0}(t),
$$

где функция $\varepsilon^{0}(\omega)$ согласно определению (32) удовлетворяет соотношениям Крамерса-Кронига (29). 
Следовательно, для ДП $\varepsilon(\omega)$ соотношения Крамерса-Кронига принимают вид

$$
\begin{aligned}
& \operatorname{Re} \varepsilon(\omega)=1+\frac{1}{\pi} \mathrm{P} \int_{-\infty}^{\infty} d \xi \frac{\operatorname{Im} \varepsilon(\xi)-4 \pi f_{\varepsilon}(\infty) / \xi}{\xi-\omega}, \\
& \operatorname{Im} \varepsilon(\omega)-\frac{4 \pi f_{\varepsilon}(\infty)}{\omega}=-\frac{1}{\pi} \mathrm{P} \int_{-\infty}^{\infty} d \xi \frac{\operatorname{Re} \varepsilon(\xi)-1}{\xi-\omega}
\end{aligned}
$$

которые полностью соответствуют соотношениям Крамерса-Кронига для ДП $\varepsilon(\omega)$ проводников (см., например, [30]), если статическая проводимость определяется равенством [33]

$$
\sigma_{\mathrm{st}}=f_{\varepsilon}(\infty)=\lim _{t \rightarrow \infty} \frac{i}{3 \hbar V}\left\langle\left[P^{\beta}(0), P^{\beta}(t)\right]\right\rangle,
$$

где переход к пределу $t \rightarrow \infty$ осуществляется после термодинамического предельного перехода.

Равенство (34) означает, что согласно определению (9) для статической проводимости $\sigma_{\text {st }}$ предел $\omega \rightarrow 0$ в формуле Кубо (24) соответствует значению этого предела под знаком временно́го интеграла. При этом функция $\varepsilon^{0}(\omega)(32)$ не имеет особенностей в пределе $\omega \rightarrow 0$.

\section{4. ОСОБЕННОСТИ ЧАСТОТНОЙ ДИСПЕРСИИ ДП ПРИ НАЛИЧИИ ОДНОЧАСТИЧНОГО КБЭ}

Используем представленные выше результаты для анализа частотной дисперсии ДП КС с одночастичным КБЭ в слабом внешнем электромагнитном поле. Предполагается, что выражения для функции $\varepsilon^{0}(\omega)(32)$ и статической проводимости $\sigma_{\text {st }}(34)$ имеют место не только для нормальной $\mathrm{KC}$, но и при наличии одночастичного КБЭ.

В этом случае согласно (18)-(20), (32) имеем

$$
\varepsilon(\omega)=\varepsilon^{0}(\omega)-\frac{\omega_{\mathrm{p}}^{2}-\Omega_{\mathrm{p}}^{2}}{\omega^{2}}+\frac{4 \pi i}{\omega} \sigma_{\mathrm{st}} .
$$

При этом функция $\varepsilon^{0}(\omega)(32)$ при наличии одночастичного КБЭ не имеет особенностей в пределе $\omega \rightarrow 0$.

Подставляя (21) в (19) и учитывая определения (3)-(6), получаем

$$
\Omega_{\mathrm{p}}^{2}=\omega_{\mathrm{p}}^{2}-\omega_{\mathrm{BEC}}^{2}, \quad \omega_{\mathrm{BEC}}^{2}=\frac{4 \pi z_{\mathrm{c}}^{2} e^{2} n_{\mathrm{c}}^{\mathrm{BEC}}}{m_{\mathrm{c}}} .
$$

Здесь учтено, что электроны, будучи фермионами, не могут образовывать одночастичный КБЭ.

Подставляя (36) в (35), получаем

$$
\varepsilon(\omega)=\varepsilon^{0}(\omega)-\frac{\omega_{\mathrm{BEC}}^{2}}{\omega^{2}}+\frac{4 \pi i}{\omega} \sigma_{\mathrm{st}} .
$$

В нормальной $\mathrm{KC}$, где $n_{\mathrm{c}}^{\mathrm{BEC}}=0$, соотношение (37) переходит в (32).

Таким образом, при наличии одночастичного КБЭ для ядер, когда $n_{\mathrm{c}}^{\mathrm{BEC}} \neq 0$, ДП $\varepsilon(\omega)$ имеет особенность второго порядка при $\omega \rightarrow 0$,

$$
\left.\varepsilon(\omega)\right|_{\omega \rightarrow 0} \rightarrow-\frac{\omega_{\mathrm{BEC}}^{2}}{\omega^{2}},
$$


в отличие от ситуации в нормальной системе, где определяющей является особенность первого порядка (10). Это означает, что, в отличие от нормальной КС (9), определение статической проводимости $\sigma_{\text {st }}$ в общем случае следует записывать как

$$
\sigma_{\text {st }}=\lim _{\omega \rightarrow 0} \operatorname{Re} \sigma(\omega)>0,
$$

учитывая, что величина $\operatorname{Re} \sigma(\omega)$ характеризует поглощение энергии слабого внешнего электромагнитного поля в длинноволновом пределе в $\mathrm{KC}: \operatorname{Re} \sigma(\omega)>0$ [17], [21]. При этом соотношения Крамерса-Кронига для ДП $\varepsilon(\omega)(37)$ принимают вид

$$
\begin{aligned}
& \operatorname{Re} \varepsilon(\omega)=1-\frac{\omega_{\mathrm{BEC}}^{2}}{\omega^{2}}+\frac{1}{\pi} \mathrm{P} \int_{-\infty}^{\infty} d \xi \frac{\operatorname{Im} \varepsilon(\xi)-4 \pi \sigma_{\mathrm{st}} / \xi}{\xi-\omega}, \\
& \operatorname{Im} \varepsilon(\omega)=\frac{4 \pi \sigma_{\mathrm{st}}}{\omega}-\frac{1}{\pi} \mathrm{P} \int_{-\infty}^{\infty} d \xi \frac{\operatorname{Re} \varepsilon(\xi)-1+\omega_{\mathrm{BEC}}^{2} / \xi^{2}}{\xi-\omega} .
\end{aligned}
$$

Из соотношения (38) с учетом формул Френеля (см., например, [30]) следует, что при падении электромагнитной волны, которая характеризуется “низкой” частотой $\omega$, на границу КС с одночастичным КБЭ для ядер такая волна будет практически полностью отражаться от поверхности. Этот эффект подобен "выталкиванию" слабонеоднородного электромагнитного поля из "обычного" электронного сверхпроводника (эффект Мейсснера). Таким образом, в КС при наличии одночастичного КБЭ имеет место “сверхпроводимость ядер”.

Отметим, что полученные результаты опираются лишь на концепцию НДДП (1), точные соотношения для операторов в представлении чисел заполнения, а также на строгие выражения для ДП КС в рамках теории линейного отклика. Поэтому приведенный вывод соотношения (38) справедлив для произвольно сильного кулоновского взаимодействия электронов и ядер, которое может приводить, в частности, к образованию связанных состояний электронов и ядер, трактуемых как атомарные.

Согласно полученным результатам функция $\operatorname{Re} \varepsilon(\omega)$ при наличии одночастичного КБЭ для ядер является отрицательной величиной при малых частотах, вплоть до предельных частот $\omega \rightarrow 0$. Неравенство $\operatorname{Re} \varepsilon(\omega) \leqslant 0$ в области низких частот $\omega$ является достаточным условием для установления наличия одночастичного КБЭ для ядер в КС. Это утверждение основано на том, что полученные результаты не связаны с использованием теории возмущений по межчастичному взаимодействию, а базируются лишь на коммутационных соотношениях и общей концепции НДДП (в применении к одночастичному КБЭ для ядер). Как уже было отмечено ранее, электроны, являясь фермионами, не могут образовывать одночастичный КБЭ, но влияют на параметры перехода КС в состояние с КБЭ ядер.

Подчеркнем также, что до настоящего времени не решен принципиальный вопрос о том, является ли сверхтекучий Не-ІІ примером жидкости с одночастичным КБЭ. При этом следует иметь в виду, что в феноменологической теории сверхтекучести Ландау [34] явление сверхтекучести не связано с наличием КБЭ (см. подробнее [35]). Обсуждение вопроса о соотношении между подходами Ландау и Боголюбова при описании спонтанного нарушения симметрии представлено в работе [36].

Таким образом, появляется возможность для прямой экспериментальной проверки наличия одночастичного КБЭ в сверхтекучем Не-II. При реализации предлагаемых экспериментов необходимо учитывать, что формула (38) является предельным соотношением при $\omega \rightarrow 0$. Другими словами, в рамках предлагаемой теории 
(см. (36)) отсутствует возможность достоверного установления значения частоты $\omega$ внешнего электромагнитного поля, начиная с которой функция $\operatorname{Re} \varepsilon(\omega)$ принимает отрицательные значения.

Общий анализ результатов экспериментов [4], [5], [37], проведенный в работе [27], позволяет сделать вывод о том, что для интерпретации наблюдаемого резонансного поглощения необходимо допустить наличие таких коллективных возбуждений в сверхтекучем Не-II, которые характеризуются ненулевым значением их энергии при предельно малых импульсах. Очевидно, что именно этому условию удовлетворяют коллективные возбуждения, определяемые равенством $\operatorname{Re} \varepsilon\left(\omega_{0}\right)=0$. Это равенство соответствует общему дисперсионному уравнению, хорошо известному в теории КС в длинноволновом пределе при условии $\operatorname{Im} \varepsilon\left(\omega_{0}\right) \ll 1$ [8], [17].

Наличие такого рода коллективных возбуждений соответствует первоначальному варианту феноменологической теории сверхтекучести Ландау [34], в рамках которой, наряду с фононами, предполагалось существование в сверхтекучем Не-II элементарных возбуждений, которые характеризуются отличным от нуля значением их энергии при предельно малых импульсах.

Следует отметить, что для интерпретации экспериментально наблюдаемого резонансного поглощения в сверхтекучем Не-II к настоящему времени предложено множество различных моделей (подробная библиография по этому вопросу представлена в [38], [39]). Предлагаемый эксперимент по исследованию отражения электромагнитных волн от поверхности сверхтекучего Не-II позволит однозначно решить вопрос о наличии одночастичного КБЭ в сверхтекучем Не-ІІ. При этом согласно экспериментальным данным по статической проводимости [40] сверхтекучий Не-II является "хорошим" диэлектриком. Однако необходимо учитывать, что состояние сверхпроводимости может быть "разрушено" при определенной величине напряженности электромагнитного поля [41].

Возможный отрицательный результат подобного эксперимента будет означать, что сверхтекучий Не-ІІ не является жидкостью с одночастичным КБЭ. В этой связи заметим, что при описании сверхтекучего Не-II может быть использовано представление о парном (двухчастичном) КБЭ, который может возникнуть в результате достаточно сильного эффективного притяжения между бозонами (см. [3] и цитированную там литературу). Такое представление аналогично КБЭ для куперовских пар в теории электронной сверхпроводимости [41], но выходит за рамки проведенного рассмотрения.

\section{Список литературы}

[1] Е. М. Лифшиц, Л. П. Питаевский, Статистическая физика. Ч. 2. Теория конденсированного состояния, Наука, М., 1978.

[2] B. V. Svistunov, E.S. Babaev, N.V. Prokof'ev, Superfluid States of Matter, CRC Press, Boca Raton, 2015.

[3] E. A. Pashitskii, S. V. Mashkevich, S. I. Vilchynskyy, "Superfluid Bose liquid with a suppressed BEC and an intensive pair coherent condensate as a model of ${ }^{4} \mathrm{He}$ ", Phys. Rev. Lett., 89:7 (2002), 075301, 4 pp.

[4] А. С. Рыбалко, "Наблюдение электрической индукции, обусловленной волной второго звука в Не II", ФНT, 30:12 (2004), 1321-1325. 
[5] A. Rybalko, S. Rubets, E. Rudavskii, V. Tikhly, S. Tarapov, R. Golovashchenko, V. Derkach, "Resonance absorption of microwaves in He II: evidence for roton emission", Phys. Rev. B, 76:14 (2007), 140503, 4 pp.

[6] Э. А. Пашицкий, С. М. Рябченко, "О причине электрической активности сверхтекучего гелия при возбуждении в нем волн второго звука и колебаний скорости нормальной компоненты", ФНТ, 33:12 (2007), 12-21.

[7] С. И. Шевченко, А.С. Рукин, "Об электрической активности сверхтекучих систем", Писъма в ЖЭЭТФ, 90:1 (2009), 46-50.

[8] В.-Д. Крефт, Д. Кремп, В. Эбелинг, Г. Рёпке, Квантовая статистика систем заряженных частии, Мир, М., 1988.

[9] J. M. McMahon, M. A. Morales, C. Pierleoni, D. M. Ceperley, "The properties of hydrogen and helium under extreme conditions", Rev. Modern Phys., 84:4 (2012), 1607-1653.

[10] А. В. Буренин, "О значении приближения Борна-Оппенгеймера во внутримолекулярной динамике", УФН, 180:7 (2010), 745-757.

[11] В. Б. Бобров, "О статистической теории разреженного газа в кулоновской модели вещества. Адиабатическое приближение и исходные атомы”, ТМФ, 178:3 (2014), 433-448.

[12] В. Б. Бобров, С. А. Тригер, "On the properties of systems with Bose-Einstein condensate in the Coulomb model of matter", Краткие сообщения по физике ФИАН, 42:1 (2015), $58-64$.

[13] O. Penrose, L. Onsager, "Bose-Einstein condensation and liquid helium", Phys. Rev., 104:3 (1956), 576-584.

[14] C. N. Yang, "Concept of off-diagonal long-range order and the quantum phases of liquid He and of superconductors", Rev. Modern Phys., 34:4 (1962), 694-704.

[15] Н. Н. Боголюбов, Н. Н. Боголюбов (мл.), Введение в квантовую статистическую механику, Наука, М., 1984.

[16] В.Б. Бобров, А. Г. Загородний, С. А. Тригер, "Недиагональный дальний порядок и неоднородный конденсат Бозе-Эйнштейна", Докл. PA H, 461:4 (2015), 400-402.

[17] В.П. Силин, А. А. Рухадзе, Электромагнитные свойства плазмы и плазмоподобных сред, Госатомиздат, М., 1961.

[18] H. Reinholz, R. Redmer, G. Röpke, A. Wierling, "Long-wavelength limit of the dynamical local-field factor and dynamical conductivity of a two-component plasma", Phys. Rev. E, 62:4 (2000), 5648-5666.

[19] H. Reinholz, Yu. Zaporoghets, V. Mintsev, V. Fortov, I. Morozov, G. Röpke, "Frequency-dependent reflectivity of shock-compressed xenon plasmas", Phys. Rev. E, 68:3 (2003), 036403, 10 pp.

[20] P. C. Martin, "Sum rules, Kramers-Kronig relations, and transport coefficients in charged systems", Phys. Rev., 161:1 (1967), 143-155.

[21] Д. Н. Зубарев, Неравновесная статистическая термодинамика, Наука, М., 1971.

[22] Л. Д. Ландау, Е. М. Лифшиц, Курс теоретической физики, т. 5: Статистическая физика. Часть 1, Наука, М., 1976.

[23] А. А. Абрикосов, Л. П. Горьков, И. Е. Дзялошинский, Методы квантовой теории поля в статистической физике, Физматгиз, М., 1962.

[24] V.B. Bobrov, V.D. Ozrin, S. A. Trigger, "Some peculiarities of the long-wavelength conductivity limit in a charged particle system", Phys. A, 164:2 (1990), 453-468.

[25] В. Б. Бобров, Н. И. Ключников, С. А. Тригер, “Точные соотношения для структурного фактора кулоновской системы", ТМФ, 89:2 (1991), 263-277.

[26] Е. М. Лифшиц, Л. П. Питаевский, Курс теоретической физики, т. 10: Физическая кинетика, Наука, М., 1979.

[27] V.B. Bobrov, S. A. Trigger, A. G. Zagorodny, "Kubo formula for frequency dispersion of dielectric permittivity and static conductivity of the Coulomb system", Phys. Lett. A, 375:2 (2010), 84-87. 
[28] R. Kubo, "Statistical-mechanical theory of irreversible processes. I. General theory and simple applications to magnetic and conduction problems", J. Phys. Soc. Japan, 12:6 (1957), 570-586.

[29] V. Ambegoaker, W. Kohn, "Electromagnetic properties of insulators. I.", Phys. Rev., 117:2 (1960), 423-431.

[30] Л. Д. Ландау, Е. М. Лифшиц, Курс теоретической физики, т. 8: Электродинамика сплошных сред, Наука, М., 1982.

[31] V.B. Bobrov, S. A. Trigger, "The true dielectric and ideal conductor in the theory of the dielectric function of the Coulomb system", J. Phys. A: Math. Theor., 43:36 (2010), 365002, $11 \mathrm{pp}$.

[32] V.B. Bobrov, "Features of the dielectric permittivity of the Coulomb system and the true dielectric state", Phys. Rev. E, 86:2 (2012), 026401, 4 pp.

[33] V. B. Bobrov, S. A. Trigger, G. J. F. van Heijst, P. P. J. M. Schram, "Kramers-Kronig relations for the dielectric function and the static conductivity of Coulomb systems", Europhys. Lett., 90:1 (2010), 10003, 4 pp.

[34] Л. Д. Ландау, “Теория сверхтекучести гелия IІ”, ЖЭТФ, 11:6 (1941), 592-624.

[35] Е. М. Лифшиц, “Теория сверхтекучести гелия II”, УФН, 34:4 (1948), 512-559.

[36] Д.В. Ширков, “Облики симметрии в современной физике”, ТМФ, 170:2 (2012), 292-303.

[37] А. С. Рыбалко, С. П. Рубец, Э. Я. Рудавский, В. А. Тихий, Ю. М. Полуэктов, Р. В. Головащенко, В.Н. Деркач, С.И. Тарапов, О.В. Усатенко, "Резонансное возбуждение единичных ротонов в Не II электромагнитной волной. Контур спектральной линии", ФНT, 35:11 (2009), 1073-1080.

[38] Ю.М.Полуэктов, "Поглощение энергии электромагнитного поля сверхтекучей системой атомов с дипольным моментом", ФНT, 40:5 (2014), 503-512.

[39] В. Б. Бобров, А. Г. Загородний, С. А. Тригер, "Кулоновский потенциал взаимодействия и конденсат Бозе-Эйнштейна", ФНТ, 41:11 (2015), 1154-1163.

[40] M. Wolfke, W.H. Keesom, "On the electrical resistance of liquid helium", Physica, 3:8 (1936), 823-824.

[41] J. R. Schrieffer, Theory of Superconductivity, Perseus Books, Reading, MA, 1999.

Поступила в редакцию 3.10 .2016 , после доработки 13.1.2017 